\title{
A NOTE ON TWO SUMMABILITY METHODS
}

\author{
W. T. SULAIMAN
}

\begin{abstract}
A theorem connecting the two summabilities $\left|\bar{N}, p_{n}\right|_{k}$ and $|C, \alpha|_{k}$ is proved. This theorem contains as special cases the result of Mohapatra 1967.
\end{abstract}

\section{Introduction}

Let $\Sigma a_{n}$ be an infinite series of partial sums $s_{n}$. Let $\sigma_{n}^{\delta}$ and $\eta_{n}^{\delta}$ denote the $n$th Cesaro mean of order $\delta(\delta>-1)$ of the sequences $\left\{s_{n}\right\}$ and $\left\{n a_{n}\right\}$ respectively. The series $\Sigma a_{n}$ is said to be absolutely summable $(C, \delta)$ with index $k$, or simply summable $|C, \delta|_{k}, k \geq 1$, if

$$
\sum_{n=1}^{\infty} n^{k-1}\left|\sigma_{n}^{\delta}-\sigma_{n-1}^{\delta}\right|^{k}<\infty,
$$

or equivalently

$$
\sum_{n=1}^{\infty} \frac{1}{n}\left|\eta_{n}\right|^{k}<\infty
$$

Let $\left\{p_{n}\right\}$ be a sequence of positive real constants such that

$$
P_{n}=\sum_{v=0}^{n} p_{v} \rightarrow \infty \text { as } n \rightarrow \infty \quad\left(P_{-1}=p_{-1}=0\right) .
$$

A series $\Sigma a_{n}$ is said to be summable $\left|\bar{N}, p_{n}\right|_{k}, k \geq 1$, if

$$
\sum_{n=1}^{\infty}\left(\frac{p_{n}}{p_{n}}\right)^{k-1}\left|T_{n}-T_{n-1}\right|^{k}<\infty \quad \text { (Bor 1986) }
$$

Received. June 4, 1990. 
where

$$
T_{n}=\frac{1}{P_{n}} \sum_{v=0}^{n} p_{v} s_{v}
$$

For $p_{n}=\frac{1}{n+1}$, the summability $\left|\bar{N}, p_{n}\right|$, which is the same as $\left|\bar{N}, p_{n}\right|_{1}$, is equivalent to the summability $|R, \log n, 1|$, and for $p_{n}=1,\left|\bar{N}, p_{n}\right|_{k}$ summability is equivalent to $|C, 1|_{k}$ summability. In general the two summability methods $|C, \delta|_{k}$ and $\left|\bar{N}, p_{n}\right|_{k}$ are independent of each other.

The following results are known:

Theorem A (Mohapatra 1967) Let sequence $\left\{\epsilon_{n}\right\}$ satisfy the following

$$
\begin{gathered}
\epsilon_{n}=o(1) \quad \text { as } n \rightarrow \infty \\
\sum_{n=1}^{\infty} n^{-\alpha+1} \lambda_{n} \log (n+1)\left|\triangle \epsilon_{n}\right|<\infty \quad(0<\alpha \leq 1) \\
\sum_{n=1}^{\infty} \lambda_{n} \log (n+1)\left|\triangle \epsilon_{n}\right|<\infty \quad(\alpha>1) ; \\
\sum_{n=1}^{\infty} \lambda_{n} n \log (n+1)\left|\triangle^{2} \epsilon_{n}\right|<\infty ;
\end{gathered}
$$

where $\left\{\lambda_{n}\right\}$ is a positive non-diminishing sequence of $n$. Then $\sum a_{n} \epsilon_{n}$ is summable $|C, \alpha|(\alpha>0)$, whenever

$$
\sum_{n=1}^{m}\left|T_{n}-T_{n-1}\right|=0 \quad\left(\lambda_{m}\right)
$$

We prove the following

Theorem 1. Let $\left\{p_{n}\right\}$ be a sequence of positive numbers such that $n p_{n}=$ $O\left(P_{n}\right)$ and $\left\{P_{n} / n p_{n}\right\}$ non-decreasing. Let $\left\{\epsilon_{n}\right\}$ be such that $\epsilon_{n}=o(1)$. Let $\left\{\lambda_{n}\right\}$ be positive non-decreasing such that

$$
\sum_{n=1}^{m}\left(\frac{P_{n}}{p_{n}}\right)^{k-1}\left|\Delta T_{n-1}\right|^{k}=O\left(\lambda_{m}^{k}\right), \quad m \rightarrow \infty
$$


where $T_{n}$ is the $\left(\bar{N}, p_{n}\right)$-mean of the $\sum a_{n}$. If

$$
\begin{aligned}
& \sum_{n=1}^{\infty} n^{k-k \alpha-1} \frac{P_{n}}{p_{n}}\left|\triangle \epsilon_{n}\right| \lambda_{n}^{k}<\infty \quad(0<\alpha<1) \\
& \sum_{n=1}^{\infty} \frac{1}{n} \frac{P_{n}}{p_{n}}\left|\triangle \epsilon_{n}\right| \lambda_{n}^{k}<\infty \quad(\alpha \geq 1)
\end{aligned}
$$

and

$$
\sum_{n=1}^{\infty} \frac{P_{n}}{p_{n}}\left|\triangle^{2} \epsilon_{n}\right| \lambda_{n}^{k}<\infty
$$

then the series $\sum a_{n} \epsilon_{n}$ is summable $|C, \alpha|_{k}, k \geq 1, \alpha>0$.

Remark. If we put $p_{n}=\frac{1}{n+1}, k=1$ in Theorem 1 , we obtain Theorem A.

\section{Lemma}

We required the following Lemma for the proof of the Theorem Lemma [3] - If $\sigma>\delta>0$, then

$$
\sum_{n=v+1}^{m} \frac{(n-v)^{\delta-1}}{n^{\sigma}}=O\left(v^{\delta-\sigma}\right), \quad m \rightarrow \infty .
$$

\section{Proof of Theorem 1}

Let $t_{n}^{\alpha}$ be the $n$th $(C, \alpha)$-mean, $\alpha>0$, of the sequence $\left\{n a_{n} \in_{n}\right\}$. Then we have

$$
\begin{gathered}
t_{n}^{\alpha}=\frac{1}{A_{n}^{\alpha}} \sum_{v=1}^{n} A_{n-v}^{\alpha-1} v a_{v} \in_{v}, \quad T_{n}-T_{n-1}=\frac{P_{n}}{P_{n} P_{n-1}} \sum_{v=1}^{n} P_{v-1} a_{v} . \\
t_{n}^{\alpha}=\frac{1}{A_{n}^{\alpha}} \sum_{v=1}^{n} P_{v-1} a_{v}\left\{v A_{n-v}^{\alpha-1} P_{v-1}^{-1} \epsilon_{v}\right\} \\
=\frac{1}{A_{n}^{\alpha}}\left[\sum_{v=1}^{n-1}\left\{\sum_{r=1}^{v} P_{r-1} a_{r}\right\} \triangle_{v}\left\{v A_{n-v}^{\alpha-1} P_{v-1}^{-1} \epsilon_{v}\right\}+\left\{\sum_{v=1}^{n} P_{v-1} a_{v}\right\} n P_{n-1}^{-1} \epsilon_{n}\right]
\end{gathered}
$$




$$
\begin{aligned}
= & \frac{1}{A_{n}^{\alpha}}\left[\sum _ { v = 1 } ^ { n - 1 } \left\{v A_{n-v}^{\alpha-1} \epsilon_{v} \Delta T_{v-1}+\frac{P_{v-1}}{P_{v}} A_{n-v}^{\alpha-1} \epsilon_{v} \Delta T_{v-1}+(v+1) \frac{P_{v-1}}{P_{v}}\right.\right. \\
& \left.\left.\Delta_{v} A_{n-v}^{\alpha-1} \epsilon_{v} \Delta T_{v-1}+(v+1) \frac{P_{v-1}}{P_{v}} A_{n-v-1}^{\alpha-1} \Delta \epsilon_{v} \Delta T_{v-1}\right\}+n \frac{P_{n}}{P_{n}} \epsilon_{n} \Delta T_{n-1}\right] \\
= & t_{n, 1}^{\alpha}+t_{n, 2}^{\alpha}+t_{n, 3}^{\alpha}+t_{n, 4}^{\alpha}+t_{n, 5}^{\alpha}, \quad \text { say. }
\end{aligned}
$$

In order to prove the Theorem, by Minkowski's inequality, it is therefore sufficient to show that

$$
\sum_{n=1}^{\infty} \frac{1}{n}\left|t_{n, r}^{\alpha}\right|^{k}<\infty, \quad r=1,2,3,4,5 .
$$

Applying Hölder's inequality,

$$
\begin{aligned}
\sum_{n=2}^{m+1} \frac{1}{n}\left|t_{n, 1}^{\alpha}\right|^{k} & \leq \sum_{n=2}^{m+1} \frac{1}{n A_{n}^{\alpha}} \sum_{v=1}^{n-1} v^{k} A_{n-v}^{\alpha-1}\left|\epsilon_{v}\right|^{k}\left|\Delta T_{v-1}\right|^{k}\left\{\sum_{v=1}^{n-1} \frac{A_{n-v}^{\alpha-1}}{A_{n}^{\alpha}}\right\}^{k-1} \\
& =O(1) \sum_{v=1}^{m} v^{k}\left|\epsilon_{v}\right|^{k}\left|\Delta T_{v-1}\right|^{k} \sum_{n=v+1}^{m+1} \frac{A_{n-v}^{\alpha-1}}{n A_{n}^{\alpha}} \\
& =O(1) \sum_{v=1}^{m} v^{k}\left|\epsilon_{v}\right|^{k}\left|\Delta T_{v-1}\right|^{k} \sum_{n=v+1}^{m+1} \frac{(n-v)^{\alpha-1}}{n^{1+\alpha}} \\
& =O(1) \sum_{v=1}^{m} v^{k-1}\left|\epsilon_{v}\right|\left|\Delta T_{v-1}\right|^{k} \\
& =O(1) \sum_{v=1}^{\infty}\left(\frac{P_{v}}{p_{v}}\right)^{k-1}\left|\Delta T_{v-1}\right|^{k}\left|\sum_{n=v}^{\infty} \Delta \epsilon_{n}\right| \\
& =O(1) \sum_{v=1}^{\infty}\left(\frac{P_{v}}{p_{v}}\right)^{k-1}\left|\Delta T_{v-1}\right|^{k} \sum_{n=v}^{\infty}\left|\triangle \epsilon_{n}\right| \\
& =O(1) \sum_{n=1}^{\infty}\left|\Delta \epsilon_{n}\right| \sum_{v=1}^{n}\left(\frac{P_{v}}{p_{v}}\right)^{k-1}\left|\Delta T_{v-1}\right|^{k} \\
& =O(1) \sum_{n=1}^{\infty}\left|\triangle \epsilon_{n}\right| \lambda_{n}^{k} \frac{P_{n}}{p_{n}}\left|\Delta \epsilon_{n}\right| \lambda_{n}^{k}
\end{aligned}
$$




$$
\begin{aligned}
\sum_{n=2}^{m+1} \frac{1}{n}\left|t_{n, 2}^{\alpha}\right|^{k} & \leq \sum_{n=2}^{m+1} \frac{1}{n A_{n}^{\alpha}} \sum_{v=1}^{n-1}\left(\frac{P_{v}}{p_{v}}\right)^{k} A_{n-v}^{\alpha-1}\left|\epsilon_{v}\right|^{k}\left|\Delta T_{v-1}\right|^{k}\left\{\sum_{v=1}^{n-1} \frac{A_{n-v}^{\alpha-1}}{A_{n}^{\alpha}}\right\}^{k-1} \\
& =O(1) \sum_{v=1}^{m}\left(\frac{P_{v}}{p_{v}}\right)^{k}\left|\epsilon_{v}\right|^{k}\left|\Delta T_{v-1}\right|^{k} \sum_{v=1}^{n-1} \frac{A_{n-v}^{\alpha-1}}{n A_{n}^{\alpha}} \\
& =O(1) \sum_{v=1}^{m} \frac{1}{v}\left(\frac{P_{v}}{p_{v}}\right)^{k}\left|\epsilon_{v}\right|^{k}\left|\Delta T_{v-1}\right|^{k} \\
& =O(1) \sum_{v=1}^{\infty} \frac{1}{v}\left(\frac{P_{v}}{p_{v}}\right)^{k}\left|\Delta T_{v-1}\right|^{k} \sum_{n=v}^{\infty}\left|\triangle \epsilon_{n}\right| \\
& =O(1) \sum_{n=1}^{\infty}\left|\triangle \epsilon_{n}\right| \sum_{v=1}^{n} \frac{P_{v}}{v p_{v}}\left(\frac{P_{v}}{p_{v}}\right)^{k-1}\left|\Delta T_{v-1}\right|^{k} \\
& =O(1) \sum_{n=1}^{\infty} \frac{1}{n} \frac{P_{n}}{p_{n}}\left|\triangle \epsilon_{n}\right| \sum_{v=1}^{n}\left(\frac{P_{v}}{p_{v}}\right)^{k-1}\left|\Delta T_{v-1}\right|^{k} \\
& =O(1) \sum_{n=1}^{\infty} \frac{1}{n} \frac{P_{n}}{p_{n}}\left|\triangle \epsilon_{n}\right| \lambda_{n}^{k}
\end{aligned}
$$

$$
\begin{aligned}
\sum_{n=2}^{m+1} \frac{1}{n}\left|t_{n, 4}^{\alpha}\right|^{k} & \leq \sum_{n=2}^{m+1} \frac{1}{n\left(A_{n}^{\alpha}\right)^{k}} \sum_{v=1}^{n-1}(v+1)^{k}\left(\frac{P_{v}}{p_{v}}\right)^{k}\left(A_{n-v-1}^{\alpha-1}\right)^{k}\left|\Delta \epsilon_{v}\right|\left\{\sum_{v=1}^{n-1} \mid\right. \\
& \left.\times \Delta \epsilon_{v} \mid\right\}^{k-1} \\
& =O(1) \sum_{v=1}^{m} v^{k}\left(\frac{P_{v}}{p_{v}}\right)^{k}\left|\Delta \epsilon_{v}\right|\left|\Delta T_{v-1}\right|^{k} \sum_{n=v+1}^{m+1} \frac{\left(A_{n-v-1}^{\alpha-1}\right)^{k}}{n\left(A_{n}^{\alpha}\right)^{k}} \\
& =O(1) \sum_{v=1}^{m} v^{k}\left(\frac{P_{v}}{p_{v}}\right)^{k}\left|\Delta \epsilon_{v}\right|\left|\Delta T_{v-1}\right|^{k} \sum_{n=v+1}^{m+1} \frac{(n-v)^{k \alpha-k}}{n^{1+k \alpha}} \\
& =O(1) \sum_{v=1}^{m}\left(\frac{P_{v}}{p_{v}}\right)^{k}\left|\triangle \epsilon_{v}\right|\left|\Delta T_{v-1}\right|^{k} \\
& =O(1) \sum_{v=1}^{\infty}\left(\frac{P_{v}}{p_{v}}\right)^{k}\left|\Delta T_{v-1}\right|^{k}\left|\sum_{n=v}^{\infty} \Delta^{2} \epsilon_{n}\right| \\
& =O(1) \sum_{v=1}^{\infty}\left(\frac{P_{v}}{p_{v}}\right)^{k}\left|\triangle T_{v-1}\right|^{k} \sum_{n=v}^{\infty}\left|\triangle^{2} \epsilon_{n}\right| \\
& =O(1) \sum_{n=1}^{\infty}\left|\triangle^{2} \epsilon_{n}\right| \sum_{v=1}^{n}\left(\frac{P_{v}}{p_{v}}\right)^{k}\left|\triangle^{2} T_{v-1}\right|^{k}
\end{aligned}
$$




$$
\begin{aligned}
& =O(1) \sum_{n=1}^{\infty} \frac{P_{n}}{p_{n}}\left|\Delta^{2} \epsilon_{n}\right| \sum_{v=1}^{n}\left(\frac{P_{v}}{p_{v}}\right)^{k-1}\left|\Delta T_{v-1}\right|^{k} \\
& =O(1) \sum_{n=1}^{\infty} \frac{P_{n}}{p_{n}}\left|\triangle^{2} \epsilon_{n}\right| \lambda_{n}^{k}
\end{aligned}
$$

For $t_{n, 3}^{\alpha}$, we have to consider the two cases $0<\alpha<1 \& \alpha \geq 1$. For $0<\alpha<1$, we obtain

$$
\begin{aligned}
& \sum_{n=2}^{m+1} \frac{1}{n}\left|t_{n, 3}^{\alpha}\right|^{k} \\
\leq & \sum_{n=2}^{m+1} \frac{1}{n\left(A_{n}^{\alpha}\right)^{k}} \sum_{v=1}^{n-1}(v+1)^{k}\left(\frac{P_{v}}{p_{v}}\right)^{k}\left|\Delta_{v} A_{n-v}^{\alpha-1}\right|\left|\epsilon_{v}\right|^{k}\left|\Delta T_{v-1}\right|^{k}\left\{\sum_{v=1}^{n-1}\left|\Delta_{v} A_{n-v}^{\alpha-1}\right|\right\}^{k-1} \\
= & O(1) \sum_{n=2}^{m+1} \frac{1}{n^{1+k}} \sum_{v=1}^{n-1} v^{k}\left(\frac{P_{v}}{p_{v}}\right)^{k}(n-v)^{\alpha-2}\left|\epsilon_{v}\right|^{k}\left|\Delta T_{v-1}\right|^{k} \\
\times & \left\{\sum_{v=1}^{n-1}(n-v)^{\alpha-2}\right\}^{k-1} \\
= & O(1) \sum_{v=1}^{m} v^{k}\left(\frac{P_{v}}{p_{v}}\right)^{k}\left|\epsilon_{v}\right|^{k}\left|\Delta T_{v-1}\right|^{k} \sum_{n=v+1}^{m+1} \frac{(n-v)^{\alpha-2}}{n n^{1+k \alpha}} \\
= & O(1) \sum_{v=1}^{m} v^{k-k \alpha-1}\left(\frac{P_{v}}{p_{v}}\right)^{k}\left|\epsilon_{v}\right|^{k}\left|\Delta T_{v-1}\right|^{k} \\
= & O(1) \sum_{v=1}^{\infty} v^{k-k \alpha-1}\left(\frac{P_{v}}{p_{v}}\right)^{k}\left|\Delta T_{v-1}\right|^{k} \sum_{n=v}^{\infty}\left|\Delta \epsilon_{n}\right| \\
= & O(1) \sum_{n=1}^{\infty}\left|\Delta \in_{n}\right| \sum_{v=1}^{n} v^{k-k \alpha} \frac{P_{v}}{v p_{v}}\left(\frac{P_{v}}{p_{v}}\right)^{k-1}\left|\Delta T_{v-1}\right|^{k} \\
n^{k-k \alpha-1} \frac{P_{n}}{p_{n}}\left|\Delta \epsilon_{n}\right| \lambda_{n}^{k} & \\
= &
\end{aligned}
$$

When $\alpha=1, t_{n, 3}^{\alpha}=0$ as $\Delta A_{n-v}^{\alpha-1}=0$, then we are assuming $\alpha>1$, and hence

$$
\begin{aligned}
& \sum_{n=2}^{m+1} \frac{1}{n}\left|t_{n, 3}^{\alpha}\right|^{k} \\
= & O(1) \sum_{n=2}^{m+1} \frac{1}{n^{1+k^{\alpha}}} \sum_{v=1}^{n-1} v^{k}\left(\frac{P_{v}}{p_{v}}\right)^{k}(n-v)^{\alpha-2}\left|\epsilon_{v}\right|^{k}\left|\Delta T_{v-1}\right|^{k} \cdot n^{(\alpha-1)(k-1)}
\end{aligned}
$$




$$
\begin{aligned}
\left(\text { as } \sum_{v=1}^{n-1}(n-v)^{\alpha-2}\right. & \left.=O(1) \int_{1}^{n-1}(n-x)^{\alpha-2} d x=O\left(n^{\alpha-1}\right) .\right) \\
& =O(1) \sum_{v=1}^{m} v^{k}\left(\frac{P_{v}}{p_{v}}\right)^{k}\left|\epsilon_{v}\right|^{k}\left|\Delta T_{v-1}\right|^{k} \sum_{n=v+1}^{m+1} \frac{(n-v)^{\alpha-2}}{n^{k+\alpha}} \\
& =O(1) \sum_{v=1}^{m} \frac{1}{v}\left(\frac{P_{v}}{p_{v}}\right)^{k}\left|\epsilon_{v}\right|^{k}\left|\Delta T_{v-1}\right|^{k} . \\
& =O(1) \sum_{n=1}^{\infty} \frac{1}{n} \frac{P_{n}}{p_{n}}\left|\triangle \epsilon_{n}\right| \lambda_{n}^{k}, \quad \text { as in the case of } t_{n, 2}^{\alpha} .
\end{aligned}
$$

Finally,

$$
\begin{aligned}
\sum_{n=2}^{m+1} \frac{1}{n}\left|t_{n, 5}^{\alpha}\right|^{k} & =\left\{\begin{array}{l}
O(1) \sum_{n=1}^{\infty} n^{k-k \alpha-1}\left(\frac{P_{n}}{p_{n}}\right)^{k}\left|\epsilon_{n}\right|^{k}\left|\Delta T_{n-1}\right|^{k} \quad(0<\alpha<1) \\
O(1) \sum_{n=1}^{\infty} \frac{1}{n}\left(\frac{P_{n}}{p_{n}}\right)^{k}\left|\epsilon_{n}\right|^{k}\left|\Delta T_{n-1}\right|^{k} \quad(\alpha \geq 1)
\end{array}\right. \\
& =\left\{\begin{array}{l}
O(1) \sum_{n=1}^{\infty} n^{k-k \alpha-1} \frac{P_{n}}{p_{n}}\left|\Delta \epsilon_{n}\right| \lambda_{n}^{k} \quad(0<\alpha<1) \\
O(1) \sum_{n=1}^{\infty} \frac{1}{n} \frac{P_{n}}{p_{n}}\left|\Delta \epsilon_{n}\right| \lambda_{n}^{k} \quad(\alpha \geq 1),
\end{array}\right.
\end{aligned}
$$

as in the case of $t_{n, 3}^{\alpha}$.

This completes the proof of the Theorem.

\section{References}

[1] H. Bor, "A note on two summability methods", Proceedings of the American Mathematical Society, 98 (1986), 81-84.

[2] R. N. Mohapatra, "A note on summability factors", Journal of the Indian Mathematical Society, 31 (1967), 213-224.

[3] W. T. Sulaiman, "Multipliers for the $\varphi-|C, \alpha|_{k}$ summability of infinite series", Pure Appl. Math. Sci., to appear.

Dept. of Applied Sciences, College of Technological Studies, P. O. Box 42325, 70654 Kuwait. 